\title{
To what extent can mathematical modeling inform the design of clinical trials? The example of safe dose reduction of tyrosine kinase inhibitors in responding patients with chronic myeloid leukemia
}

\author{
Joshua T. Schiffer ${ }^{1,2}$ and Charles A. Schiffer ${ }^{3}$ \\ ${ }^{1}$ Vaccine and Infectious Diseases and Clinical Research Divisions, Fred Hutchinson Cancer Research Center, Seattle, WA; ${ }^{2}$ Department \\ of Medicine, University of Washington, Seattle, WA and ${ }^{3}$ Department of Oncology Wayne State University School of Medicine, \\ Karmanos Cancer Institute, Detroit, MI, USA
}

E-mail: schiffer@karmanos.org

doi:10.3324/haematol.2018.201897

T he development of the tyrosine kinase inhibitors (TKIs) that inhibit the BCR/ABL oncoprotein driving the growth and persistence of chronic myeloid leukemia (CML) is one of the most remarkable advances in anti-cancer treatment in recent decades, and has served as the model, if not the Platonic ideal, for targeted therapies of other cancers. It remains standard practice to administer TKIs indefinitely because of concern about relapse should compliance be erratic or therapy stopped. However, a number of recent trials have demonstrated that approximately $50 \%$ of patients whose transcript levels were either extremely low or undetectable for at least 2-3 years using sensitive polymerase chain reaction (PCR) assays, have not relapsed after therapy was stopped with many patients relapse free for more than five years after discontinuation. ${ }^{1,2}$ Remarkably, almost all relapses occurred within the first 68 months after therapy cessation. The mechanism(s) by which CML remains dormant is not known, although immunological explanations are proposed, and the only predictors of continued remission are longer durations of TKI therapy and PCR negativity.

The benefits of these so-called "treatment-free remissions $^{\prime \prime 3}$ are obvious, and include substantially reduced costs (CML-directed TKIs also served as the prototype for the obscene pricing of "targeted" agents), ${ }^{4}$ decreases in the potential for serious longer term organ toxicities (fortunately very rare with imatinib ${ }^{5}$ ), elimination of the often bothersome, low-grade chronic side-effects such as fatigue and diarrhea, as well as the ability to plan for pregnancies in younger patients. But, it is estimated that less than $20 \%$ of patients who are started on TKI treatment will be able to successfully discontinue therapy. It is likely, however, that some of these same benefits would be achieved if it were possible to reduce the dose of the TKIs without loss of disease control. Indeed, the selection of doses and schedule for many drugs is often based on very small, under-powered trials with relatively few subsequent attempts to determine whether lower doses might result in similar outcomes. In this regard, it was recently shown in a large phase II trial in newly diagnosed patients in chronic phase that a starting dose of $50 \mathrm{mg}$ of dasatinib seems to produce the same response rate as the "standard" dose of $100 \mathrm{mg}$ with perhaps fewer side-effects. ${ }^{6,7}$

In this issue of the Journal, Fassoni et al. propose what they consider to be a safe strategy of dose reduction of TKIs for CML patients in chronic phase based on mathematical models generated from large recently generated clinical trial data. ${ }^{8}$ The authors evaluated patients who were in major molecular response (MMR) for at least one year, defined as a greater than 3 log reduction in transcripts from baseline, and who had received imatinib therapy for more than three years. Their model concludes that, for most patients, halving the dose of the TKI will maintain the current level of response, albeit with a high likelihood of a temporary increase in transcript levels which return to baseline after continued treatment with the reduced dose.

Fassoni et al.'s paper ${ }^{8}$ highlights the crucial role that mathematical models can play in providing a mechanistic underpinning of observed data, and in influencing therapeutic strategies. As a general principle, differential equationbased models are a necessary tool to capture non-linearity in biomedical datasets. CML treatment involves the coupling of mechanisms governing tumor cell division, transition rates between progenitor cells and terminally differentiated tumor cells, anti-tumor immune responses and the selective pressure of TKIs. The non-linearity inherent in a model which captures the dynamics of this system can yield counterintuitive and useful predictions, such as Fassoni et al.'s calculation that the increase in BCR/ABL levels that may occur following TKI dose reduction is transitory. ${ }^{8}$ Fassoni et al. hypothesize that an increase in transcripts is due to a self-limited increase in proliferating leukemia stem cells (LSCs), rather than mutations leading to TKI resistance or changes in the underlying dynamics of quiescent, non-proliferating LSCs. ${ }^{8}$

Mathematical modeling as a field would benefit from some demystification. Well-designed models represent nothing more than in silico experiments where models with competing structures and mechanistic assumptions are compared for their ability to fit to observed data. Fassoni et al.'s model recapitulates data from selected patients from the IRIS and CML IV trials in whom the pattern of second phase decrease in transcripts is thought to result from slow depletion of quiescent LSCs. This depletion is predicted to occur independently of TKI dose reduction because the transition from quiescent to proliferating LSC, rather than incomplete efficacy of TKIs, is the rate-limiting step for CML eradication during MMR. The model's generalizability to patients with an adequate treatment response is further supported by the scalability of its key parameters governing transitions between quiescent and proliferating LSCs across all patients. Finally, the model's main prediction, that TKI dose reduction will not usually lead to loss of MMR, is qualitatively consistent with interim analyses from the DESTINY trial, a study in which 174 participants underwent $50 \%$ dose reduction as the initial phase of a "stopping" trial. ${ }^{9}$ It is not known whether the transcript increases seen in some of these patients at the reduced dose would 
have resolved, because the protocol called for restarting therapy if MMR was lost. Thus, to implement their recommendation in a subsequent clinical trial, clinicians would have to resist the current dogma to resume the higher dose or to switch to another TKI should the levels increase.

How then should the model's enticing predictions be interpreted? Similar to in vitro models or animal models which aim to recapitulate key features of human disease, the primary role of mathematical models is to generate a hypothesis for a definitive test of concept studies in humans, rather than providing "proof" of the concept. Accordingly, mathematical model simulations of clinical trials can critically inform the design of clinical trials themselves, but should never replace them. Indeed, the fact that Fassoni et al.'s model neglects several potential reasons for treatment failure in patients following dose reduction, such as de novo loss of immunological control or TKI resistance, highlights the point that the model's novel hypotheses need to be tested.

Fassoni et al.'s simulations validate the concept that effective TKI dose reduction is biologically plausible. Moreover, the output of the model provides useful information for TKI dose selection and sampling frequency in subsequent clinical trials. Model output suggests that the amount of TKI dose reduction may be individualized, based partly on observed BCR/ABL kinetics during the initial phase of therapy, which can then be used to derive two key parameters: 1) leukemic stem cell activation; and 2) TKI efficacy. Estimation of these parameters will require frequent BCR/ABL measurements during both primary and second phases of TKI therapy, an important consideration for trial design.

Most importantly, the modeling provides informed criteria for assessing treatment failure after dose reduction, and specifically recommends that investigators should not immediately classify increases in BCR-ABL transcripts as treatment failures. Rather, the model suggests that increases in BCR-ABL ratio could be permitted for as long as a year, at which point second phase decay would be expected to occur. Again, sampling following dose reduction must be frequent enough to allow model fitting that precisely characterizes this phenomenon. A third critical parameter, the LSC proliferation rate, can only be estimated following TKI dose reduction and could theoretically be leveraged to make dose adjustments in real time. Finally, because the model predicts effect size, it could also theoretically be used to inform power calculations to project an adequate sample size for 'proof of concept' trials.

To understand the importance of these findings, one only need consider the situation in which a priori mathematical modeling is not performed, and TKI dose reduction is formally tested and labeled a failure based on what could have been only a temporary increase in BCR/ABL transcript levels. This would represent a spurious rejection of an important and valid scientific hypothesis, as well as a waste of resources. It is likely that these types of errors are not uncommon in clinical trial design, and that many could be predicted a priori with the use of strategic mathematical modeling.

\section{References}

1. Mahon FX, Réa D, Guilhot J, et al. Discontinuation of imatinib in patients with chronic myeloid leukaemia who have maintained complete molecular remission for at least 2 years: the prospective, multicentre Stop Imatinib (STIM) trial. Lancet Oncol. 2010;11(11):1029 1035.

2. Ross DM, Branford S, Seymour JF, et al. Safety and efficacy of imatinib cessation for CML patients with stable undetectable minimal residual disease: results from the TWISTER study. Blood. 2013;122(4):515-522.

3. Hughes TP, Ross DM. Moving treatment-free remission into mainstream clinical practice in CML. Blood. 2016;128(1):17-23.

4. Experts in Chronic Myeloid Leukemia. The price of drugs for chronic myeloid leukemia (CML) is a reflection of the unsustainable prices of cancer drugs: from the perspective of a large group of CML experts. Blood. 2013;121(22):4439-4442.

5. Gambacorti-Passerini C, Antolini L, Mahon FX, et al. Multicenter independent assessment of outcomes in chronic myeloid leukemia patients treated with imatinib. J Natl Cancer Inst. 2011;103(7):553-561.

6. Naqvi K, Jabbour E, Skinner J, et al. Early results of lower dose dasatinib (50 mg daily) as frontline therapy for newly diagnosed chronic-phase chronic myeloid leukemia. Cancer. 2018;124(13):2740-2747.

7. Schiffer CA. The evolution of dasatinib dosage over the years and its relevance to other anticancer medications. Cancer. 2018;124(13):26872689.

8. Fassoni AC, Baldow C, Roeder I, Glauche I. Reduced tyrosine kinase inhibitor dose is predicted to be as effective as standard dose in chronic myeloid leukemia: a simulation study based on phase III trial data. Hematologica. 2018 Jun 28. [Epub ahead of print]

9. Clark RE, Polydoros F, Apperley JF, et al. De-escalation of tyrosine kinase inhibitor dose in patients with chronic myeloid leukaemia with stable major molecular response (DESTINY): an interim analysis of a non-randomised, phase 2 trial. Lancet Haematol. 2017;4(7): e310-e316. 